\title{
- - Nützt eine Diät bei ADHS?
}

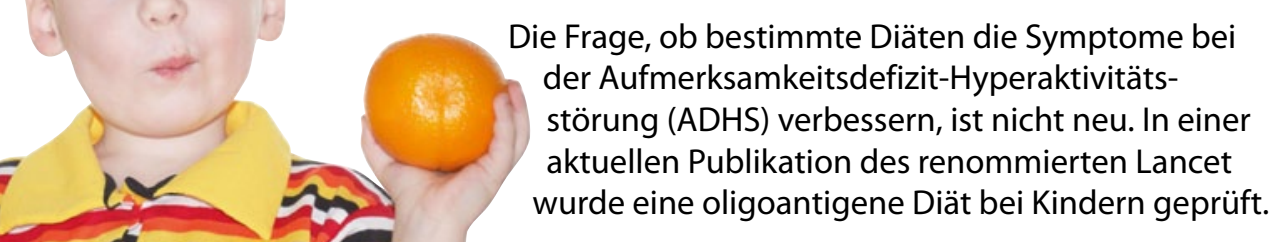

Zunächst einmal stellt sich die Frage, wie sicher die Ergebnisse sind. Es handelt sich

In der Vorstellung, dass ADHS durch eine Nahrungsintoleranz oder -hyperreagibilität entsteht oder beeinflusst wird, haben die Autoren eine zweiphasige randomisierte Studie durchgeführt. 50 Kinder mit ADHS im Alter von vier bis acht Jahren erhielten eine oligoantigene Diät, die mit Kartoffeln, Weizen und Früchten ergänzt wurde. 50 Kinder erhielten normales Essen.

41 Kinder aus der Diätgruppe (81\%) beendeten die erste Phase. ADHS-Symptome und oppositionelles Verhalten verbesserten sich in der Diätgruppe signifikant bei $64 \%$ dieser Patienten. In der Kontrollgruppe wurden dagegen keine Verbesserungen beobachtet.

Die Kinder, die in der ersten Phase auf die Diät gut angesprochen hatten, erhielten nun zusätzliche Nahrungsbestandteile - je nachdem, ob diese in einem Provokationstest zu einer Veränderung der IgG-Werte geführt hatten oder nicht. Unabhängig vom Wert des IgG gegen Nahrungsmittel kam es hier jedoch zu erneutem Auftreten der ADHS-Symptomatik bei Hinzufügen dieser Nahrungsbestandteile.

Zur Beurteilung der Symptomatik war bei Eltern, Erziehern und Ärzte eine ADHS-Ratingskala nach Connors verwendet worden. Eltern und Erzieher waren im Gegensatz zum Arzt nicht "geblindet“. Die Autoren schließen aus den Ergebnissen, dass eine oligoantigene Diät signifikant die ADHS-Symptomatik mindert und daher als Therapieoption zu erwägen ist.

Kommentar: In Einzelfällen wird immer wieder berichtet, dass durch Weglassen eines bestimmten Nahrungsbestandtteiles sich das Verhalten ändert. 1985 veröffentlichte Eggers seine Studie, die eine Verbesserung der Symptomatik bei einem Teil der untersuchten Kinder zeigte. Ist also generell eine oligoantigene Diät bei Kindern mit ADHS zu empfehlen? hier nicht um eine Doppelblindstudie. Das Rating des "geblindeten“ Arztes entstand durch dessen Befragung der Eltern, die nicht geblindet waren und bei denen evtl. ein Coping-Effekt vorlag. Es wird auch nichts darüber ausgesagt, wie die Patienten ausgewählt worden waren. Möglicherweise besteht auch hier ein Bias: Eltern, die eine alternative Therapie wünschen und von einer Diät überzeugt sind, werden sich eher bereit erklären, an einer sehr aufwendigen und einschneidenden Studie teilzunehmen als andere, die den diätetischen Möglichkeiten kritisch gegenüberstehen.

Aber auch wenn die Wirksamkeit einer Diät dauerhaft bestehen würde, stellt sich die Frage, ob eine länger durchgeführte Diät überhaupt mit dem praktischen Alltag vereinbar ist. Es besteht die Gefahr, dass — das betroffene Kind zum sozialen Außenseiter wird,

— es eventuell zu einer Fehlernährung kommt,

— die ganze Familie in Mitleidenschaft gezogen wird und

— die Kinder die Diät umgehen (aus der Zeit der Phosphatdiäten ist bekannt, dass das Schulbrot gerne mit dem Nachbarn getauscht wird).

Bevor generelle Empfehlungen für eine oligoantige Diät ausgesprochen werden, halte ich es daher für sinnvoll, die Studienlage zu verbessern, Langzeitdaten und Langzeit-Nebenwirkungen zu evaluieren. Es sind auch Kriterien zu etablieren, welche Kinder von einer Diät profitieren könnten. Entsprechend den Ergebnissen der vorliegenden Studie würden immerhin knapp $40 \%$ der Kinder mit ADHS keine Wirkung auf eine Diät zeigen. Dr. Kirsten Stollhoff

Pelsser LM et al. Effects of a restricted elimination diet on the behaviour of children with attention-deficit hyperactivity disorder (INCA study): a randomised controlled trial. Lancet 2011; 377: 494-503 